This is the final peer-reviewed accepted manuscript of:

Matteucci, M., Veldkamp, B.P. The approach of power priors for ability estimation in IRT models. Qual Quant 49, 917-926 (2015). https://doi.org/10.1007/s11135014-0059-y

The final published version is available online at: https://doi.org/10.1007/s11135$\underline{014-0059-y}$

Rights / License:

The terms and conditions for the reuse of this version of the manuscript are specified in the publishing policy. For all terms of use and more information see the publisher's website. 


\title{
The approach of power priors for ability estimation in IRT models
}

\author{
Mariagiulia Matteucci • Bernard P. \\ Veldkamp
}

Received: date / Accepted: date

\begin{abstract}
The aim of the paper is to propose the introduction of power prior distributions in the ability estimation of item response theory (IRT) models. In the literature, power priors have been proposed to integrate information coming from historical data with current data within Bayesian parameter estimation for generalized linear models. This approach allows to use a weighted posterior distribution based on the historical study as prior distribution for the parameters in the current study. Applications can be found especially in clinical trials and survival studies. Here, power priors are introduced within a Gibbs sampler scheme in the ability estimation step for a unidimensional IRT model. A Markov chain Monte Carlo algorithm is chosen for the high flexibility and possibility of extension to more complex models. The efficiency of the approach is demonstrated in terms of measurement precision by using data from the Hospital Anxiety and Depression Scale (HADS) with a small sample.
\end{abstract}

Keywords Power priors · Item response theory models · Ability estimation · Gibbs sampler

\author{
M. Matteucci \\ Department of Statistical Sciences, University of Bologna \\ via Belle Arti, 4140126 Bologna, Italy \\ Tel.: +39-0541434051 \\ Fax: +39-051232153 \\ E-mail: m.matteucci@unibo.it \\ B.P. Veldkamp \\ Research Center for Examination and Certification, University of Twente \\ P.O. Box 217, 7500 AE Enschede, Netherlands \\ E-mail: b.p..veldkamp@gw.utwente.nl
}




\section{Introduction}

In Bayesian estimation, where posterior evidence is derived from the combination of the likelihood and a prior distribution, the elicitation of the prior remains a crucial issue. Although non-informative priors can be employed in absence of prior information (see [14]), they can cause instability in the posterior estimates and convergence problems for the Gibbs sampler ([13]). In applied research, prior information on the parameters of interests is often available in the form of historical data, collateral information or predictions from subject matter experts. In order to combine historical data with observed data, power prior distributions were proposed for the parameter estimation of regression models ([13]) and more generally of generalized linear models ([6]). This approach allows to discount historical data by introducing an appropriate weight. Generally, the introduction of informative priors is strongly encouraged with small sample sizes, in case the available data provide only indirect information about the parameters of interests, and for preventing from inappropriate inferences, consistent with the likelihood (see [11]).

The availability of a small sample is also a recurring context in educational and psychological measurement, where item response theory (IRT) models ([15]) are commonly used. Given the responses of a sample of subjects to categorical test items, IRT models express the probability of an item response as a function of the item psychometric properties and the latent abilities underlying the response process. Therefore, parameter estimation involves two phases: (a) calibration, where the item parameters are estimated, and (b) scoring, where the subjects are located on the latent trait scale. In both phases, the issue of measurement precision is very important, especially with small samples. In fact, a large number of respondents is needed for calibration and, conversely, as many items as possible are needed for an accurate ability estimation. As a rule of thumb, for a two-parameter unidimensional model, a calibration sample size of at least 500 subjects and 20 or more items is suggested (see [7], page 105). Obviously, in practice it is not always possible to rely on optimal testing conditions. For this reason, the introduction of collateral or historical information in model estimation assumes a particular importance and one possibility for improving the parameter estimation would be to include informative prior distributions.

Bayesian estimation of IRT models through Markov chain Monte Carlo (MCMC) has become very popular recently (see, among others, [3]; [10]; [21]; [19]) because it allows the simultaneous estimation of item parameters and individual abilities while overcoming multiple integration problems of marginal maximum likelihood (MML) estimation and model limitations of conditional maximum likelihood (CML) estimation (for a review of both methods in IRT, see [23]). Under this approach, both item parameters and abilities are viewed as random variables, with a corresponding prior distribution. Therefore, it is easily possible to introduce informative priors, both empirical or not empirical, to increase measurement precision and compensate for a small sample size. In particular, the capability of taking uncertainty into account, i.e. to treat the 
item parameters not as fixed quantities, is fundamental ([24]). Further advantages are the possibility of using Bayesian model comparison techniques, such as Bayes factors, Bayesian deviance and posterior predictive model checks (for a recent study see [2]). Despite the use of MCMC was limited in the past due to its computational intensity, the increasing availability of cheap computing power stimulated its present diffusion. The introduction of random item models, where the items are assumed to be random draws from a population with a specific distribution, is rather recent. This approach was found to be effective in measuring longitudinal ability change, explanation of item difficulties and studies on differential item functioning (see [4] and [8]).

In order to increase the measurement precision, the introduction of empirical prior information was proposed by capitalizing on the relationship between the parameters of interests and a set of auxiliary variables (e.g. [22]; [17]; [18]; [16]). However, historical data such as data on previous testing rounds or previous test administrations, consisting in the item responses, the estimated item parameters and abilities of respondents, can also be introduced in the prior distributions.

For these reasons, we propose the introduction of historical data through the approach of power priors for ability estimation in the unidimensional twoparameter normal ogive (2PNO) IRT model. The power prior distribution is defined directly in the Gibbs sampler step performing the ability sampling. As a case study, we take into account response data collected through the administration of the Hospital Anxiety and Depression Scale (HADS) questionnaire to a sample of patients.

The paper is organized as follows. Section 2 gives a brief overview of the approach of power priors. In Section 3, MCMC estimation for IRT models is reviewed taking into account the $2 \mathrm{PNO}$ model, and the new proposal of introducing the power prior distribution in the ability sampling step is presented. Section 4 discusses the application of the approach to real data while concluding remarks end the paper.

\section{Power priors}

The underlying idea of power priors is to introduce information coming from historical data in the estimation of the model parameters based on current data. According to Ibrahim and Chen ([13]), historical data are defined as data arising from previous similar studies, where the same response variable and covariates of the current study have been collected. In applied research, and especially in medical research, it is very common that similar studies are conducted repeatedly. For example, in clinical trials, data on similar or slightly modified treatments are often available. Also previous data collected in longitudinal studies can be classified as historical data, and could be used to compare and interpret the results based on current data.

Given the current data $D=(n, \mathbf{y}, \mathbf{X})$, where $n, \mathbf{y}$, and $\mathbf{X}$ are the sample size, the vector of dependent variables, and the matrix of covariates, respec- 
tively, the likelihood function for the current study can be expressed generally by $\mathcal{L}(\theta \mid D)$ with $\theta$ representing the parameters of interests. Analogously, let $D_{0}=\left(n_{0}, \mathbf{y}_{0}, \mathbf{X}_{0}\right)$, be the historical data. Given the initial prior distribution $\pi_{0}(\theta \mid \cdot)$ for $\theta$ before the historical data are observed, the power prior distribution of $\theta$ for the current study can be defined as

$$
\pi\left(\theta \mid D_{0}, a_{0}\right) \propto \mathcal{L}\left(\theta \mid D_{0}\right)^{a_{0}} \pi_{0}\left(\theta \mid c_{0}\right),
$$

where $0 \leq a_{0} \leq 1$ is a scalar parameter controlling for the influence of the historical data on the current data, and $c_{0}$ is the hyperparameter of the initial prior $([13])$. According to this definition, the power prior distribution is the product of the likelihood function based on the historical data, raised to a power $a_{0}$, and the initial prior distribution for $\theta$. The parameter $a_{0}$ is a relative precision parameter for the historical data which controls the heaviness of the tails for $\pi\left(\theta \mid D_{0}, a_{0}\right)$, in fact as $a_{0}$ becomes smaller, the tails become heavier. Two limit cases can be distinguished: $a_{0}=1$ where the prior of the current study corresponds to the posterior of the previous study, and $a_{0}=0$ where no historical data are incorporated.

By following an hierarchical approach, it is also possible to specify a proper prior distribution for the precision parameter $a_{0}$ in order to obtain the so called joint power prior distribution, as follows

$$
\pi\left(\theta, a_{0} \mid D_{0}\right) \propto \mathcal{L}\left(\theta \mid D_{0}\right)^{a_{0}} \pi_{0}\left(\theta \mid c_{0}\right) \pi\left(a_{0} \mid \gamma_{0}\right),
$$

where $\gamma_{0}$ is a vector of hyperparameters. There are several choices for the prior distribution for $a_{0}$, such as beta, truncated gamma and truncated normal distributions ([13]). Starting from the initial idea of Diaconis and Ylvisaker $([9])$, power priors have been developed for regression models $([13])$, and more generally for generalized linear models $([6])$.

\section{Bayesian estimation via MCMC of IRT models}

IRT models (see, e.g., [15]) allow the analysis of categorical response data through the assumption of one or more latent variables underlying the response process. Given a set of $k$ categorial items submitted to a sample of $n$ subjects, the model is designed to infer the psychometric characteristics of test items and the individual traits. Depending on the number of model parameters and the latent dimensionality, these models can be very complex making the classical estimation based on MML rather difficult due to multiple integration problems. An alternative approach is represented by simulation techniques such as MCMC, a general class of methods where the posterior distribution of interest is reproduced through the simulation of one or more sequences of correlated random variables. Basically, the advantages of MCMC are the simultaneous estimation of item parameters and candidate scores, the incorporation of the dependencies among variables and all sources of uncertainties and the possibility of using Bayesian model comparison techniques. A 
practical problem of MCMC is represented by the computational intensity of the method. However, thanks to the increasing availability of cheap computing power, this obstacle is going to be removed, even for large data sets.

In the IRT literature, within the MCMC methods, the Gibbs sampler ([12]) was introduced for the estimation of the unidimensional $2 \mathrm{PNO}$ model by Albert ([1]). The $2 \mathrm{PNO}$ model is chosen because of its feasibility within Bayesian estimation, in fact the probit link function allows to use normal-normal conjugate families. In the literature, the method was extended to, for example, the implementation of the Gibbs sampler for the estimation of multidimensional IRT models ([3]), multilevel IRT models ([10]) and IRT models with general and specific latent traits $([21])$.

In the following, the general algorithm for the estimation of the $2 \mathrm{PNO}$ model is described and a modification of the Gibbs sampler step for ability estimation is proposed in order to introduce the power prior.

\subsection{Gibbs sampler for the 2PNO model}

Under the assumption of unidimensionality, i.e. the existence of a single latent ability underlying the response process, given a binary response variable $Y_{i j}$ for individual $i$ to item $j$, where $Y_{i j}=1$ for a correct response and $Y_{i j}=0$ for an incorrect response, with $i=1, \ldots, n$ and $j=1, \ldots, k$, the 2PNO model ([15]) specifies the probability of a correct response as a monotonically increasing function of the trait, as follows

$$
P\left(Y_{i j}=1 \mid \theta_{i}, \alpha_{j}, \delta_{j}\right)=\Phi\left(\alpha_{j} \theta_{i}-\delta_{j}\right)=\int_{-\infty}^{\alpha_{j} \theta_{i}-\delta_{j}} \frac{1}{\sqrt{2 \pi}} e^{-z^{2} / 2} d z,
$$

where $\theta_{i}$ is the ability of person $i, \alpha_{j}$ and $\delta_{j}$ are the item parameters for item $j$, and $\Phi$ is the cumulative normal distribution function. In particular, the discrimination parameter $\alpha_{j}$ assesses the power of the item to differentiate respondents of different ability, while the difficulty parameter $\delta_{j}$ represents the threshold or difficulty level of the item. Theoretically, discriminations could take all values in the real line. However, positive values are found in practice, ensuring that the probability of endorsing an item increases as ability increases. Difficulties can take values in the set of real numbers and a mean and median value around zero would ensure a balanced pool between easy and difficult items in the test.

In order to be used within the Gibbs sampler, the dichotomous response variables $Y_{i j}$ should be modeled as indicators of the corresponding underlying variables $Z_{i j}$, which are defined to be conditionally independent and identically distributed as $Z_{i j} \sim N\left(\eta_{i j} ; 1\right)$, where $\eta_{i j}=\alpha_{j} \theta_{i}-\delta_{j}$. Given a standard normal prior distribution for $\theta_{i}$, i.e. $\theta_{i}$ i.i.d. $\sim N(0,1)$, and an indicator function of positiveness for discrimination parameters as prior distribution for the item parameters $\boldsymbol{\xi}$, i.e. $P(\boldsymbol{\xi})=\prod_{j=1}^{k} I\left(\alpha_{j}>0\right)$, where $\boldsymbol{\xi}$ is the vector containing 
the $k$ elements $\boldsymbol{\xi}_{j}=\left(\alpha_{j}, \delta_{j}\right)$, the joint posterior distribution (see [1]) can be expressed as

$$
\begin{aligned}
P(\mathbf{Z}, \boldsymbol{\theta}, \boldsymbol{\xi} \mid \mathbf{Y})= & P(\mathbf{Z} \mid \boldsymbol{\theta}, \boldsymbol{\xi}, \mathbf{Y}) P(\boldsymbol{\theta}) P(\boldsymbol{\xi}) \\
\propto & \prod_{i=1}^{n} \prod_{j=1}^{k}\left\{\phi\left(Z_{i j} ; \eta_{i j}, 1\right)\left[I\left(Z_{i j}>0\right) I\left(y_{i j}=1\right)+I\left(Z_{i j} \leq 0\right) I\left(y_{i j}=0\right)\right]\right\} \\
& \prod_{i=1}^{n} \phi\left(\theta_{i} ; 0,1\right) \prod_{j=1}^{k} I\left(\alpha_{j}>0\right) .
\end{aligned}
$$

Distribution (4) has an intractable form, so we should resort to the Gibbs sampler. The algorithm is applied in order to iteratively sample from the following conditional distributions, given $\mathbf{Y}$, until convergence:

\section{1. $\mathbf{Z} \mid \boldsymbol{\theta}, \boldsymbol{\xi}$ \\ 2. $\boldsymbol{\theta} \mid \mathbf{Z}, \boldsymbol{\xi}$ \\ 3. $\boldsymbol{\xi} \mid \boldsymbol{\theta}, \mathbf{Z}$.}

The first conditional distribution of the $Z_{i j}$ is truncated normal, as follows

$$
Z_{i j} \mid \boldsymbol{\theta}, \boldsymbol{\xi} \sim\left\{\begin{array}{l}
N\left(\eta_{i j}, 1\right) \text { with } Z_{i j}>0 \text { if } Y_{i j}=1, \\
N\left(\eta_{i j}, 1\right) \text { with } Z_{i j} \leq 0 \text { if } Y_{i j}=0 .
\end{array}\right.
$$

The second conditional distribution is obtained by starting from the normal regression model $Z_{i j}+\delta_{j}=\alpha_{j} \theta_{i}+\epsilon_{i j}$, where $\epsilon_{i j}$ i.i.d. $\sim N(0,1)$ and $\theta_{i}$ is viewed as regression coefficient. Therefore, the likelihood function of $\theta_{i}$ follows the normal distribution with mean equal to the least square estimate of $\theta_{i}$, specifically $\hat{\theta}_{i}=\sum_{j=1}^{k} \alpha_{j}\left(Z_{i j}+\delta_{j}\right) / \sum_{j=1}^{k} \alpha_{j}^{2}$, and variance $v=1 / \sum_{j=1}^{k} \alpha_{j}^{2}$. According to the standard normal prior imposed on $\theta_{i}$, the posterior distribution turns out to be normal and parameterized as

$$
\theta_{i} \mid \mathbf{Z}, \boldsymbol{\xi} \sim N\left(\frac{\hat{\theta}_{i} / v}{1 / v+1}, \frac{1}{1 / v+1}\right) .
$$

The third conditional distribution can be derived by following the same approach and considering the normal regression model $\mathbf{Z}_{j}=\mathbf{X} \boldsymbol{\xi}_{j}+\boldsymbol{\epsilon}_{j}$, where $\mathbf{X}=$ $[\boldsymbol{\theta}-\mathbf{1}], \boldsymbol{\epsilon}_{\boldsymbol{j}}$ is a random sample from $N(0 ; 1), \boldsymbol{\xi}_{\boldsymbol{j}}$ are viewed as regression coefficients. Given a multivariate normal likelihood $\mathbf{Z}_{j} \mid \boldsymbol{\xi}_{j} \sim N\left(\left(\mathbf{X}^{\prime} \mathbf{X}\right)^{-1} \mathbf{X}^{\prime} \mathbf{Z}_{j} ;\left(\mathbf{X}^{\prime} \mathbf{X}\right)^{-1}\right)$, a vague prior $P(\boldsymbol{\xi})=\prod_{j=1}^{k} I\left(\alpha_{j}>0\right)$ turns out with the following posterior distribution for the item parameters

$$
\boldsymbol{\xi}_{j} \mid \mathbf{Z}, \boldsymbol{\theta} \sim N\left(\left(\mathbf{X}^{\prime} \mathbf{X}\right)^{-1}\left(\mathbf{X}^{\prime} \mathbf{Z}_{j}\right) ;\left(\mathbf{X}^{\prime} \mathbf{X}\right)^{-1}\right) I\left(\alpha_{j}>0\right) .
$$

Alternatively, by using a normal prior distribution for both discrimination and threshold parameters $\alpha_{j} \sim N\left(0 ; \sigma_{\alpha}^{2}\right), \delta_{j} \sim N\left(0 ; \sigma_{\delta}^{2}\right)([3])$, the corresponding posterior distribution becomes 


$$
\boldsymbol{\xi}_{\boldsymbol{j}} \mid \mathbf{Z}, \boldsymbol{\theta} \sim N\left(\left(\mathbf{X}^{\prime} \mathbf{X}+\boldsymbol{\Sigma}_{\mathbf{0}}^{-1}\right)^{-1}\left(\mathbf{X}^{\prime} \mathbf{Z}_{j}\right) ;\left(\mathbf{X}^{\prime} \mathbf{X}+\boldsymbol{\Sigma}_{\mathbf{0}}^{-1}\right)^{-1}\right),
$$

where $\boldsymbol{\Sigma}_{\mathbf{0}}$ is the variance-covariance matrix for the item parameters.

Starting from a set of starting values, the Gibbs sampler proceeds with the iterative sampling from the conditional distributions until convergence. The choice of starting values is not crucial in MCMC but reasonable initial points may speed convergence.

\subsection{Ability estimation for the $2 \mathrm{PNO}$ model with power priors}

The introduction of a power prior distribution for the ability requires a modification of the second conditional distribution within the Gibbs sampler described in Sect. 3.1. First of all, recall that the conditional distribution for $\theta_{i}$ derives from the regression model $Z_{i j}+\delta_{j}=\alpha_{j} \theta_{i}+\epsilon_{i j}$, where $\theta_{i}$ is treated as regression coefficient. Our historical data consist of $D_{0}=\left(k_{0}, Z_{i j 0}+\delta_{j 0}, \alpha_{j 0}\right)$, where $k_{0}$ is the number of items in the historical study, $Z_{i j 0}+\delta_{j 0}$ and $\alpha_{j 0}$ are the observed values for the dependent variable and the regressor, respectively.

Given the scalar precision parameter $a_{0}$, a general formulation for the power prior is $\pi\left(\theta_{i} \mid D_{0}, a_{0}\right) \propto L\left(\theta_{i} \mid D_{0}\right)^{a_{0}} \pi_{0}\left(\theta_{i}\right)$. The likelihood is again normal and specifically: $Z_{i j}+\delta_{j} \mid \theta_{i}, D_{0} \sim N\left(\hat{\theta}_{i 0}, a_{0}^{-1} v_{0}\right)$, where $\hat{\theta}_{i 0}=\sum_{j=1}^{k} \alpha_{j 0}\left(Z_{i j 0}+\right.$ $\left.\delta_{j 0}\right) / \sum_{j=1}^{k} \alpha_{j 0}^{2}$ and $v_{0}=1 / \sum_{j=1}^{k} \alpha_{j 0}^{2}$. Assuming a standard normal as initial prior $\pi_{0}\left(\theta_{i}\right)$, i.e. $\theta_{i} \sim N(0,1)$, it can be easily shown that, from its combination with the normal likelihood, the following power prior distribution for ability can be derived

$$
\theta_{i} \mid D_{0}, a_{0} \sim N\left(\frac{a_{0} \hat{\theta}_{i 0} / v_{0}}{a_{0} / v_{0}+1} ; \frac{1}{a_{0} / v_{0}+1}\right) .
$$

The last step consists in deriving the corresponding posterior distribution for ability, to be used as the conditional distribution for $\theta_{i}$ in the Gibbs sampler step. Taking into account once again the normal-normal Bayesian model, combining the power prior (9) with the normal likelihood for ability described in Sect. 3.1, the following posterior distribution can be derived after simple arithmetic manipulations

$$
\theta_{i} \mid \mathbf{Z}, \boldsymbol{\xi}, D_{0}, a_{0} \sim N\left(\left(\frac{\hat{\theta}_{i}}{v}+\frac{a_{0} \hat{\theta}_{i 0}}{v_{0}}\right)\left(\frac{1}{v}+\frac{a_{0}}{v_{0}}+1\right)^{-1} ;\left(\frac{1}{v}+\frac{a_{0}}{v_{0}}+1\right)^{-1}\right)
$$

\section{Case study}

An empirical application is presented in order to show the effectiveness of including a power prior distribution in increasing the measurement precision of the ability estimates when the sample size is small. 
Despite IRT applications are mainly in the field of educational and psychological measurement, there is an increasing interest in using these models in the medical field. In fact, data used in this study come from the administration of the well-known Hospital Anxiety and Depression Scale (HADS) questionnaire to a sample of Dutch hospital patients. The questionnaire investigates the anxiety and depression state by using a set of 14 items, which are originally scored on an ordinal scale from 0 to 3, denoting an increasing level of psychological disease. Here, the item responses were reverse scored, when needed, and dichotomized so that 0 means "lack of disease" and 1 means "presence of disease". Data consist of the item responses at a baseline measurement and four subsequent repeated measurements. The idea is to use the data at the third measurement as historical data to estimate the "ability" $\theta$ of patients for the current and last fourth measurement. In this context, ability should be interpreted as the anxiety and depression state, so that patients with an high ability estimates are associated to an high level of disease.

The sample of patients at the baseline measurement consists of $58.2 \%$ of males and $34.6 \%$ of smokers. The average age is about 72 , with the youngest patient being 52 and the oldest one being 85 years old. At baseline measurement, the average rate of negative responses meaning the presence of disease was $47.67 \%$. This rate is only slightly reduced at measurement $3(47.62 \%)$ while at measurement 4 a relevant improvement may be observed in the patients' state, in fact the rate becomes $46.17 \%$. By computing the raw score for all the measurements, a median value of 7 can be observed for the baseline and the third measurement, while a median value of 6.5 is recorded for the fourth measurement, meaning again that the psychological conditions of patients improved over time.

The $2 \mathrm{PNO}$ item parameters were estimated in the baseline measurement on a sample of $n=154$ patients. The parameter estimates are reported in Table 1.

Table 1 Item parameter estimates for the baseline measurement ( $\mathrm{Sd}=$ standard deviation)

\begin{tabular}{lllll}
\hline Item & $\hat{\alpha}_{j}$ & $\mathrm{Sd}_{\alpha}$ & $\hat{\delta}_{j}$ & $\mathrm{Sd}_{\delta}$ \\
\hline 1 & 1.25 & 0.26 & -0.70 & 0.16 \\
2 & 1.04 & 0.19 & 0.04 & 0.12 \\
3 & 0.78 & 0.16 & 0.12 & 0.11 \\
4 & 1.57 & 0.28 & 0.90 & 0.17 \\
5 & 1.35 & 0.26 & -0.31 & 0.14 \\
6 & 2.05 & 0.48 & 0.61 & 0.18 \\
7 & 1.17 & 0.23 & -0.72 & 0.15 \\
8 & 0.67 & 0.19 & -1.26 & 0.16 \\
9 & 0.90 & 0.17 & 0.33 & 0.12 \\
10 & 0.71 & 0.15 & 0.31 & 0.11 \\
11 & 1.62 & 0.32 & -0.64 & 0.16 \\
12 & 1.32 & 0.25 & -0.14 & 0.13 \\
13 & 1.31 & 0.28 & 0.72 & 0.16 \\
14 & 0.81 & 0.18 & 0.63 & 0.13 \\
\hline
\end{tabular}




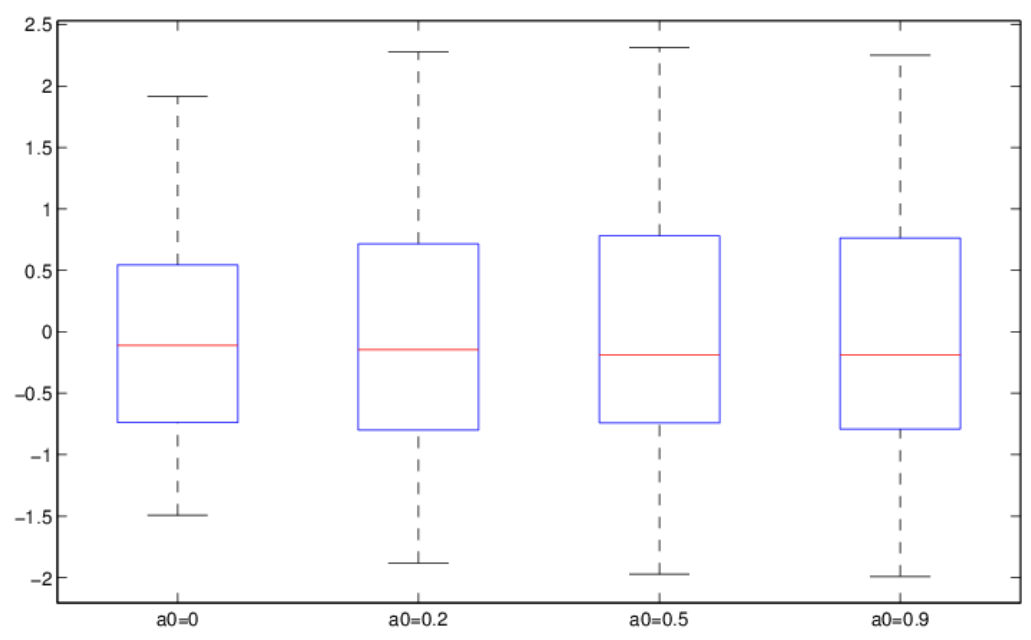

Fig. 1 Boxplots of ability estimates under different choices for $a_{0}$

The results show that the $\alpha$ parameters are all largely positive ensuring a good capability of all items to differentiate among patients with different levels of anxiety and depression. Moreover, the threshold parameters $\delta$ are rather balanced in terms of positive and negative values ensuring that the questionnaire contains items with different levels of criticity, i.e. items which are more or less likely to be answered positively by patients with different levels of anxiety and depression. The estimated item parameters in the baseline measurement are then treated as fixed over test administrations.

The focus is instead on investigating the measurement precision of the ability estimates under different approaches. For this reason, we used the Gibbs sampler under the $2 \mathrm{PNO}$ model to estimate the abilities of $n=123$ patients that responded to the HADS questionnaire at measurement 4. We introduced historical data on measurement 3 in the form of the power prior distribution (9) for ability, by specifying different precision parameters: $a_{0}=0.0, a_{0}=0.2$, $a_{0}=0.5$, and $a_{0}=0.9$. In particular, $a_{0}$ equal to zero means that historical data are not introduced in the ability estimation for the current study while, on the contrary, $a_{0}$ equal to 0.9 means an assignment of a very large prior weight to historical information. The Gibbs sampler reached the convergence within all approaches after 5000 iterations (500 burn-in). All analyses were conducted by using a Matlab program written by the Authors. The output consists on the entire posterior distribution of the ability for each patient. The estimated abilities are derived as the expected mean from the posterior distribution. Box-plots showing the distribution of the estimated abilities for all patients are reported in Fig. 1. The results clearly show that, when a prior weight is given to historical data, the range is higher meaning that it is possible to distinguish patients with different levels of the latent trait deeply. 
Table 2 Summary statistics for the standard deviation (Sd) and the $95 \%$ credibility interval (CI) width of the ability estimates under different choices for $a_{0}$

\begin{tabular}{lllll}
\hline & $a_{0}=0$ & $a_{0}=0.2$ & $a_{0}=0.5$ & $a_{0}=0.9$ \\
\hline Sd & & & & \\
Mean & 0.35 & 0.29 & 0.23 & 0.19 \\
Median & 0.32 & 0.28 & 0.23 & 0.19 \\
Min & 0.26 & 0.23 & 0.20 & 0.18 \\
Max & 0.57 & 0.40 & 0.29 & 0.22 \\
CI width & & & & \\
Mean & 1.35 & 1.12 & 0.91 & 0.76 \\
Median & 1.24 & 1.09 & 0.90 & 0.75 \\
Min & 1.01 & 0.92 & 0.80 & 0.68 \\
Max & 2.21 & 1.55 & 1.11 & 0.86 \\
\hline
\end{tabular}

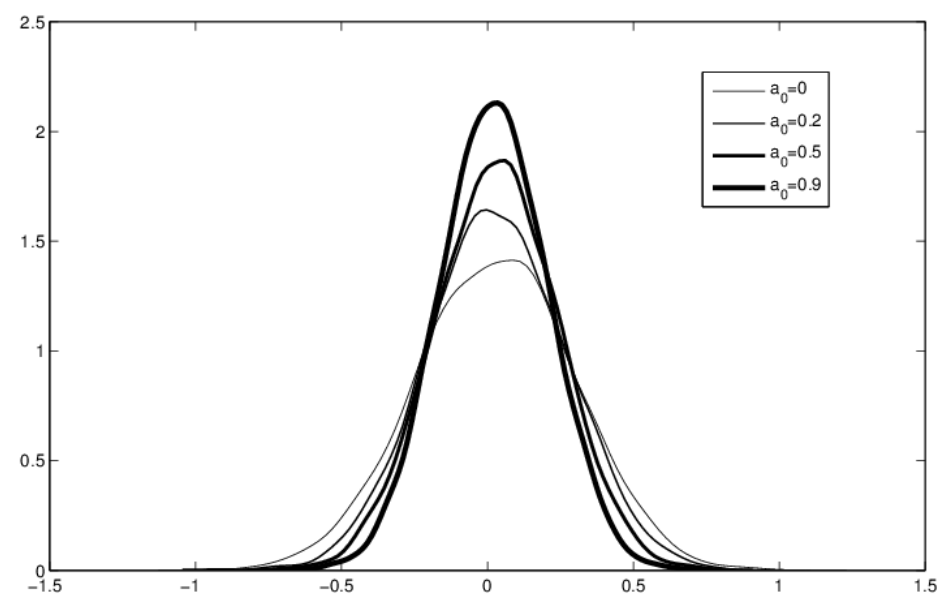

Fig. 2 Kernel density for the ability of a respondent with $\hat{\theta}=0.03$ under different choices for $a_{0}$

For each ability parameter, the expected a posteriori estimate, the standard deviation, and the $95 \%$ credibility interval were identified. In Table 2, some summary statistics on the measurement precision (standard deviation and width of the credibility interval) are reported to summarize the results.

As can be easily noticed, there is a relevant improvement in the precision of the ability estimates as the prior weight is increased. This is demonstrated by lower standard deviation and CI width on average.

Lastly, Fig. 2 reports the kernel density for the ability of a respondent with an intermediate ability which is estimated within all approaches in $\hat{\theta}=0.03$. An increase in the prior weight $a_{0}$ is represented by an increase in the thickness of the density line. The plot clearly shows that the heaviness of the tails is reduced as $a_{0}$ becomes larger and, consequently, there is a noticeable improvement in the measurement precision. The large influence of the historical data is also enhanced by the presence of a small sample $(n=123)$. 


\section{Discussion}

In this work, the introduction of a power prior distribution based on historical data is proposed for the ability estimation based on the current data in the unidimensional 2PNO IRT model. In particular, the power prior is introduced in the ability sampling step for the Gibbs sampler, within a fully Bayesian approach. The effectiveness of the method is demonstrated in a case study by using data coming from a HADS study, where the latent trait of interest is the anxiety and the depression state of hospital patients. A small sample was taken into account in order to show the potential of the power prior to improve the measurement precision when there is lacking information from the current study. The inclusion of power priors allowed to integrate coherently data coming from the current study and historical data, by adopting different weights. An increase in the importance given to the prior weight resulted in an improvement in the measurement precision of the anxiety and depression state of patients. However, we should note that the effectiveness of the approach relies on the quality of historical data.

The paper provided several insights from which draw on some further research. First of all, a sensitivity study should be conducted on different choices for the relative precision parameter $a_{0}$. In particular, elicitation of the parameter $a_{0}$ can be driven by meta-analytic arguments by using the connections between power priors and hierarchical models as underlined in [5]. An alternative approach would be to specify a joint power prior distribution for the parameter of interest and the power parameter ([13]). However, some difficulties have been highlighted in the literature with reference to the specification of a prior distribution for the $a_{0}$ parameter (see, [20]). Also, more informative initial priors could be combined in the definition of the power prior. Another relevant issue would be to study the integration of multiple sources of information in the power prior and the possibility of combining multiple historical data which can be easily available in longitudinal studies. Finally, from the IRT model point of view, it would be very important to derive an analogous approach to introduce power priors in the estimation of item parameters.

Acknowledgements This research has been partially funded by the Italian Ministry of Education with the FIRB ("Futuro in ricerca") 2012 project on "Mixture and latent variable models for causal-inference and analysis of socio-economic data". We would like to thank Prof. Job van der Palen from the University of Twente for providing us the HADS data.

\section{References}

1. Albert, J.H.: Bayesian estimation of normal ogive item response curves using Gibbs sampling. J. Educ. Stat. 17, 251-269 (1992)

2. Azevedo, C.L.N., Andrade, D.F., Fox, J-P.: A Bayesian generalized multiple group IRT model with model-fit assessment tools. Comput. Stat. Data Anal. 56, 4399-4412 (2012)

3. Béguin, A.A., Glas, C.A.W.: MCMC estimation and some model-fit analysis of multidimensional IRT models. Psychometrika 66, 541-562 (2001)

4. Briggs, D.C., Wilson, M.: Generalizability in item response modeling. J. Educ. Meas. 44, 131-155 (2007) 
5. Chen, M.H., Ibrahim, J.G.: The relationship between the power prior and hierarchical models. Bayesian Anal. 1(3), 551-574 (2006)

6. Chen, M.H., Ibrahim, J.G., Shao, Q.M.: Power prior distributions for generalized linear models. J. Stat. Plan. Inf. 84, 121-137 (2000)

7. de Ayala, R.J.: The theory and practice of item response theory. Guilford Press, New York (2009)

8. De Boeck, P.: Random item IRT models. Psychometrika 73, 533-559 (2008)

9. Diaconis, P., Ylvisaker, D.: Conjugate priors for exponential families. Ann. Stat. 7, 269$281(1979)$

10. Fox, J-P., Glas, C.A.W.: Bayesian estimation of a multilevel IRT model using Gibbs sampling. Psychometrika 66, 271-288 (2001)

11. Gelman, A. (2002). Prior distribution. In: El-Shaarawi, A.H., Piegorsch, W.W. (eds.) Encyclopedia of Environmetrics, Vol. 3, pp. 1634-1637. John Wiley \& Sons, New York (2002)

12. Geman, S., Geman, D.: Stochastic relaxation, Gibbs distributions and the Bayesian restoration of images. IEEE Trans. Pattern. Anal. Mach. Intell. 6, 721-741 (1984)

13. Ibrahim, J.G., Chen, M.H.: Power prior distributions for regression models. Stat. Sci. $15,46-60(2000)$

14. Kass, R.E., Wasserman, L.: The selection of prior distributions by formal rules. J. Am. Stat. Assoc. 91, 1343-1370 (1996)

15. Lord, F.M., Novick, M.R.: Statistical theories of mental test scores. Addison-Wesley, Reading, MA (1968)

16. Matteucci, M., Veldkamp, B.P.: Computer adaptive testing with empirical prior information: a Gibbs sampler approach for ability estimation. Stat. Meth. Appl. 22 (2), 243-267 (2013)

17. Matteucci, M., Mignani, S., Veldkamp, B.P.: Prior distributions for item parameters in IRT models. Comm. Stat. Th. Meth. 41(16-17), 2944-2958 (2012a)

18. Matteucci, M., Mignani, S., Veldkamp, B.P.: The use of predicted values for item parameters in item response theory models: an application in intelligence tests. J. Appl. Stat. 39(12), 2665-2683 (2012b)

19. Natesan, P., Limbers, C., Varni, J.W.: Bayesian estimation of graded response multilevel models using Gibbs sampling: formulation and illustration. Educ. Psych. Meas. 70(3), 420$439(2010)$

20. Neuenschwander, B., Branson, M., Spiegelhalter, D.J.: A note on the power prior. Stat. Med. 28, 3562-3566 (2009)

21. Sheng, Y., Wikle, C.K.: Bayesian IRT models incorporating general and specific abilities Behaviormetrika 36, 27-48 (2009)

22. van der Linden, W.J.: Empirical initialization of the trait estimation in adaptive testing Appl. Psych. Meas. 23, 21-29 (1999)

23. van der Linden, W.J., Hambleton, R.K.: Handbook of modern item response theory. Springer, New York (1997)

24. Veldkamp, B.P., Matteucci, M., de Jong, M.: Uncertainties in the item parameter estimates and robust automated test assembly. Appl. Psych. Meas. 37(2), 123-139 (2013) 